\title{
The integration of buses with a high level of service in the medium cities urban context
}

\author{
M. Losa, A. Pratelli \& C. Riccardi \\ Department of Civil and Industrial Engineering, University of Pisa, Italy
}

\begin{abstract}
In the last decade the world has witnessed a large diffusion of the BHLS (Buses with a High Level of Service) systems. This growth was sparked off by the successful implementation of the first BHLS system in Curitiba (Brazil) in the 1970s, also named bus rapid transit (BRT), which was able to achieve reliable and efficient transport service at reduced costs. The main reason of BHLS' spread is mostly due to its proper characteristics that allow to combine flexibility and low costs typical of bus based systems with reliability, efficiency, speed, frequency, comfort and safety offered by the more costly LRT (Light Rail Transit). These systems are generally characterized by modern environmentally friendly vehicles, reserved bus-ways and applications of intelligent transport systems (ITS) based technologies. All these characteristics are very important features of the so-called smart mobility.

The aim of this paper is to describe the working process adopted to design a new BHLS line haul in Pisa (Italy) connecting Pisa Central Railway Station with the Hospital of Cisanello. The paper shows how the new BHLS line could be fairly suited to the Italian context, and in particular to a medium sized city like Pisa, characterized by narrow streets, high density of building and need of sharing large part of bus paths in mixed traffic. Furthermore, this paper evaluates the feasibility of a solution to solve the problem concerning the give-bus priority. Signalized roundabouts with controlled traffic light and giving priority to BHLS vehicles, that allow buses to cross the intersection without stopping delays, have been proposed.

The results of such design analysis on one side confirm line haul feasibility, and on the other side show that the expected level of service of the proposed design solutions is fairly good. Finally, some brief considerations are also drawn
\end{abstract}


on BHLS transit general features giving design insights in respect to mid-size urban area requirements.

Keywords: BHLS, mid-size urban context, signalized roundabouts, priority to buses at traffic signals, smart mobility.

\section{Introduction}

The great challenge of the European community within the program "HORIZON 2020 " is to achieve a transport system that is resource-efficient, environmentally friendly, safe and seamless for the benefit of citizens, the economy and society. The purpose of EU support is to minimize transport's impact on climate and the environment by improving its efficiency in the use of natural resources, and by reducing its dependence on fossil fuels through specific objectives like reducing resource consumption and greenhouse gas emissions and improving vehicle efficiency as well as increasing the use of demand management and public transport, particularly in urban areas. Another EU objective is to reconcile growing mobility needs with improved transport fluidity, through innovative solutions for seamless, inclusive, safe, secure and robust transport systems and specific objectives like reducing congestion improving accessibility and drastically reducing the occurrence of accidents [1].

The Municipality of Pisa is moving on exactly in this way making significant investments to try to improve urban mobility building park and ride, and improving public transport through protected corridors. One of them is the new line-haul that will connect the station of Pisa Centrale to the new hospital of Cisanello, which is the focus of this paper.

Among the various types of transport systems our choice is focused on the BHLS (Bus with a High Level of Service), since it allows for the combination of flexibility and the low cost of typical bus based systems with the reliability, efficiency, speed, frequency, comfort, security typical of Light Rail Transit (LRT). In addition, the simultaneous implementation of bicycle lanes and the adoption of restrictive policies on traffic, so as to discourage the use of private vehicles in favor of the public sector, will transform the city into a more livable space.

The design of the track in dedicated lane reserved for public transport has been developed not only to improve the performance of this transport mode, but was also seized as an opportunity to retrain some important urban spaces and to extend the pedestrian and cycle network. This additional modal option with low environmental impact, in a medium-sized city showing geomorphological and urban form characteristics such as Pisa, should strongly be an effective solution for some important segments of the potential demand in the urban area and thus to contribute to the network of public transport in ensuring better environmental sustainability of all urban mobility.

Furthermore, the line-haul with all dedicated and protected lanes involves deep changes to the main road network junctions, in order to improve the overall level of service in respect to the present one. 
The strategic objectives of the project are therefore: to increase the attractiveness of the city and hence its potential demand, structuring effective transport modal option alternatives to the private car, in order to reduce the traffic flow and relieve congestion in the nodes of the road network, improving functional performance and reducing the overall impacts that the mobility system determines on the usability and livability of the city.

The aim of this work is both to describe the methodologies used to design the new connection, and to show how the proposed technologies solutions are suited for planning any roundabout embedded into a BHLS path.

\section{BHLS implementations and characteristics}

BHLS (Bus with a High Level of Service) or BRT (Bus Rapid Transit) systems were born from the need to achieve efficient and reliable transportation systems at a reduced cost to try to reduce congestion and therefore pollution of the big cities, where the private vehicle seemed to be the only means of transport to meet the needs and mobility requirements of citizens. In the last decade the world has witnessed a rapid increase of these transport systems, due to the great success of the RIT (Rede de Integrada Trasporte) of Curitiba (Brazil), that was the first BRT system to be implemented in the world (1974), and of the Trans Milenio of Bogota (Colombia), that showed as this system is able to handle very high flows of passengers over 45000 pass/h per direction. So in this big metropolis the BRT are really surface subways.

Also in Europe, for example in France, many cities decided to adopt this new type of transport system, that stands between the LRT (Light Rail Transit) and the bus based system doing appropriate adaptations to its own context: narrow streets, high density, mixed traffic lane; vehicles using innovative light guide such as the Bombardier TVR, the Civis of Irisbus, the Translohr, the Phileas.

The objectives behind the implementation of a BHLS system primarily concern organization and management of transport policies aiming at limiting the use of private vehicles, encouraging a modal shift to alternative forms of transport and at increasing satisfaction among existing public transport users. The BRT/BHLS potentials for further development is great, both in large urban areas, seeking to lend a hierarchical structure to their network, and in smaller urban areas for which BHLS represents a rapid transit system well suited to their size and capable of accompanying their urban development [2].

In France, in 2005, a workgroup heated by CERTU, defined its own concept of BRT based on initial local experience. The "new town" of Évry in the 1970s, the Trans-Val-de-Marne system for the Greater Paris region starting in 1993, TEOR in Rouen in 2001 are cited as valid instances. French concept is based on the use of on-street exclusive lanes as fundamental component allowing for the increase of speed and regularity. In the case of BHLS, its use can be limited to congested zones, such as city centers (for instance, Rouen's TEOR). BHLS is also present in the Netherlands (Amsterdam in 2001, Eindhoven in 2005), England (Leeds in 1998, Cambridge in 2009), Sweden (Gothenburg in 2003) and in Spain (Castellón in 2008). While full-BRT is not present in Europe, as it has 
not been chosen to fulfill the mass transit function, numerous systems could be classified as BRT-Lite. Among the most well known of European BHLS systems there are the blue buses of Stockholm (Sweden) since 1999, the Lianes of Dijon (France) since 2004 and the so-called LAM - Linea Alta Mobilità developed in Italy (Prato, Brescia, Pisa).

In European largest cities (over 1 million inhabitants), the subway and the tramway are already widely developed for the main arterial movements. BHLS allows for a complementary hierarchical organization of the bus network (Madrid's MBus projects, Lyon's Cristalis lines, Stockholm's blue buses, Hamburg's Metro Busse, Helsinki's Jokeri-line, and Amsterdam's Zuidtangent). In the large urban areas (300 thousand to 1 million inhabitants), BHLS can be integrated into the main rapid transit system obtaining a status equivalent to that of a tramway (BusWay in Nantes, TEOR in Rouen, Gothenburg) or it can constitute a feeder service for a heavier rapid transit system to serve the inner suburbs (exclusive bus lanes in Tolouse).

Table 1: BRT characteristics by Wright and Hook [3].

\begin{tabular}{|c|c|}
\hline BRT characteristics & BRT dimensions \\
\hline Exclusive right of way lanes & Running ways \\
\hline Convenient, secure and weather protected stations & \multirow{2}{*}{ Stations } \\
\hline $\begin{array}{l}\text { Easy access to and from other means of transport and the urban } \\
\text { environment for all groups of users }\end{array}$ & \\
\hline Rapid and comfortable boarding and alighting & \multirow{3}{*}{ Vehicles } \\
\hline High comfort vehicles & \\
\hline Low emission vehicle technologies & \\
\hline Pre board fare collection and verification & Fare collection \\
\hline Centralized system management and control & \multirow{3}{*}{$\begin{array}{l}\text { Intelligent Transport } \\
\text { System (ITS) }\end{array}$} \\
\hline Signal priority & \\
\hline Modern information facilities & \\
\hline Frequent and rapid service & \multirow{4}{*}{ Branding elements } \\
\hline A distinctive marketing identity & \\
\hline Independent quality control system & \\
\hline Excellent customer service & \\
\hline
\end{tabular}

In smaller urban areas (Castellón, Nîmes, Metz, Jönköping, Lund, Utrecht, etc.), BHLS constitutes the kernel axes of the public transportation system, allowing the renovation of urban area and creating a "rapid transit effect" that benefits the entire system/city. The analysis of different BHLS solutions and their classification in terms of role in the transport scheme, ITS applications (fleet monitoring and priority systems, users information systems, etc.) and service performance indicators have been achieved by European workgroup COST TU 0603 [2].

The specific characteristics that allow us to classify a system as a BRT have been listed by Wright and Hook [3] and are shown in Table 1. 
There is also a list of quantity indicators that define BHLS concepts proposed by CERTU [4]. An interesting classifications of these systems is shown in Table 2, that classifies the BRT systems into four differ categories: Intelligent BRT; Heavy Infrastructure BRT; Light BRT; Understatement BRT. In this tables are also indicated, for each of these categories, the unique characteristics and the correspondent rail-based system [5].

The transport system herewith presented can be defined as an Intelligent BRT as it is characterized by easily identifiable and accessible stations, dedicated lanes along the entire route, transport with optical guidance therefore constrained guide (Civis by Irisbus), ITS systems that allow to manage both traffic light priority at intersections, and all travelers information.

Table 2: Classification of BRT systems.

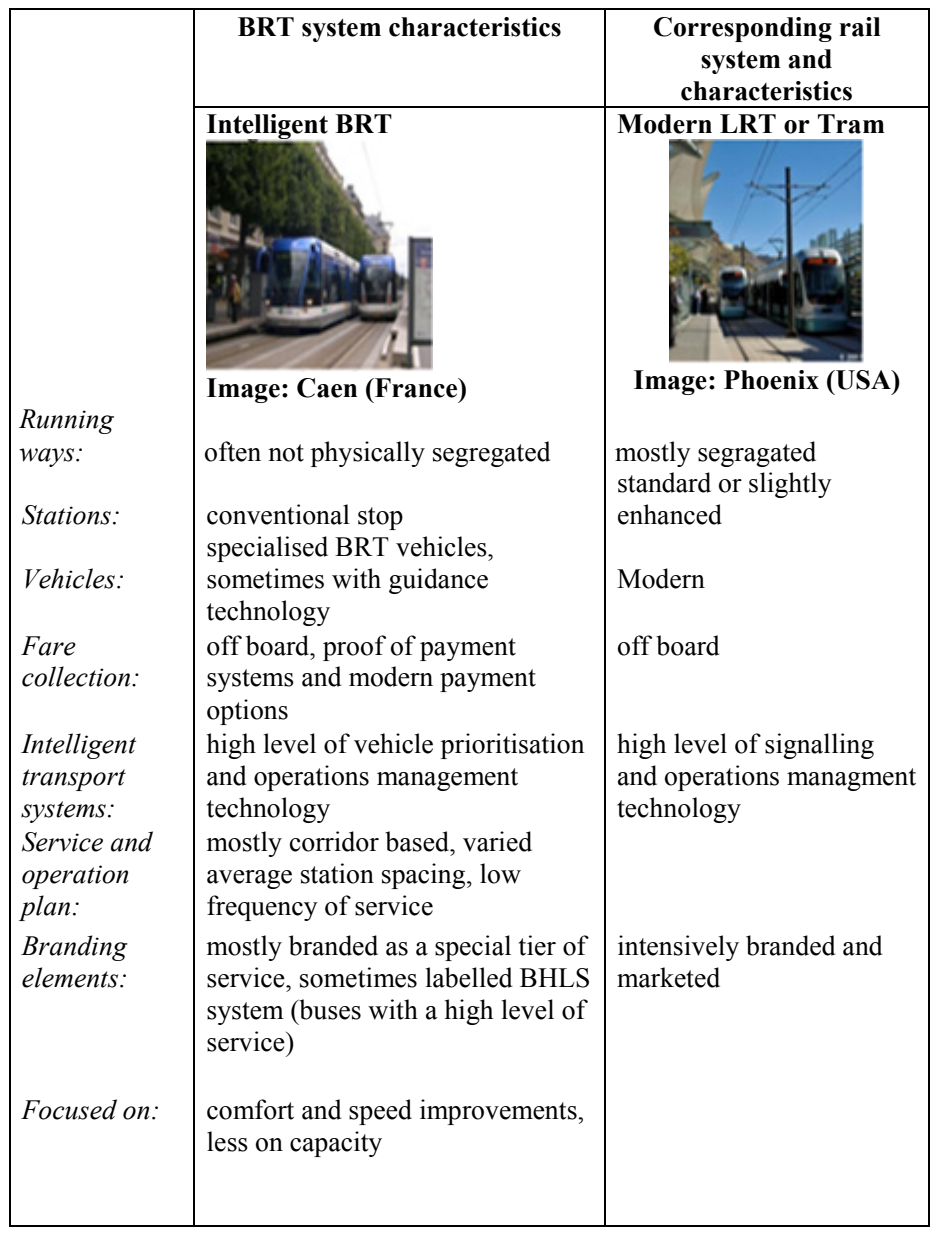


Table 2: Continued.

\begin{tabular}{|c|c|c|}
\hline & Heavy infrastructure BRT & $\begin{array}{l}\text { Segregated LRT or heavy rail or } \\
\text { sub surface metro } \\
\text { Image: Zurich (Swiss) }\end{array}$ \\
\hline Running ways: & often completely segregated & often completely segragated \\
\hline Stations: & fully enclosed, rapid boarding & $\begin{array}{l}\text { enhanced or underground stations } \\
\text { with rapid boarding and alighting }\end{array}$ \\
\hline Vehicles: & $\begin{array}{l}\text { specialised, with level access, } \\
\text { often bi-articulated buses }\end{array}$ & Specialised \\
\hline $\begin{array}{l}\text { Fare } \\
\text { collection: }\end{array}$ & $\begin{array}{l}\text { off board, barriers, often modern } \\
\text { payment options }\end{array}$ & off board \\
\hline $\begin{array}{l}\text { Intelligent } \\
\text { transport } \\
\text { systems: }\end{array}$ & $\begin{array}{l}\text { low level of vehicle prioritisation } \\
\text { and operations managment } \\
\text { technology }\end{array}$ & $\begin{array}{l}\text { high level of signalling and } \\
\text { operations managment technology }\end{array}$ \\
\hline $\begin{array}{l}\text { Service and } \\
\text { operation plan: } \\
\text { Branding } \\
\text { elements: } \\
\text { Focused on: }\end{array}$ & 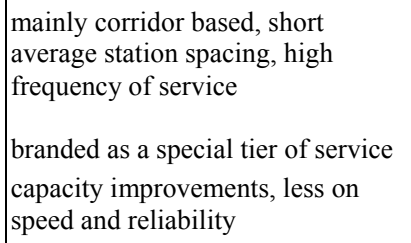 & intensively branded and marketed \\
\hline & Image: San Paolo (Brazil) & $\begin{array}{c}\text { Conventional LRT or tram } \\
\text { operation } \\
\text { Image: San Gallen (Swiss) }\end{array}$ \\
\hline Running ways & $\begin{array}{l}\text { bus lanes but no physically } \\
\text { segregated }\end{array}$ & tracks only partly segregated \\
\hline Stations & standard basic stops & standard stations \\
\hline $\begin{array}{l}\text { Vehicles } \\
\text { Fare collection }\end{array}$ & $\begin{array}{l}\text { standard or slightly enhanced } \\
\text { vehicles } \\
\text { mostly on board }\end{array}$ & $\begin{array}{l}\text { standard vehicles } \\
\text { Mostly off board }\end{array}$ \\
\hline $\begin{array}{l}\text { Intelligent } \\
\text { transport } \\
\text { systems: } \\
\text { Service and } \\
\text { operation plan: } \\
\text { Branding } \\
\text { elements } \\
\text { Focused on }\end{array}$ & $\begin{array}{l}\text { low level of vehicle prioritisation } \\
\text { and operations managment } \\
\text { technology } \\
\text { mostly network based, high to very } \\
\text { high frequency of service } \\
\text { often branded and marketed as a } \\
\text { special tier of service } \\
\text { low cost and high capacity, less on } \\
\text { reliability and comfort }\end{array}$ & $\begin{array}{l}\text { basic signalling and operations } \\
\text { management technology }\end{array}$ \\
\hline
\end{tabular}


Table 2: Continued.

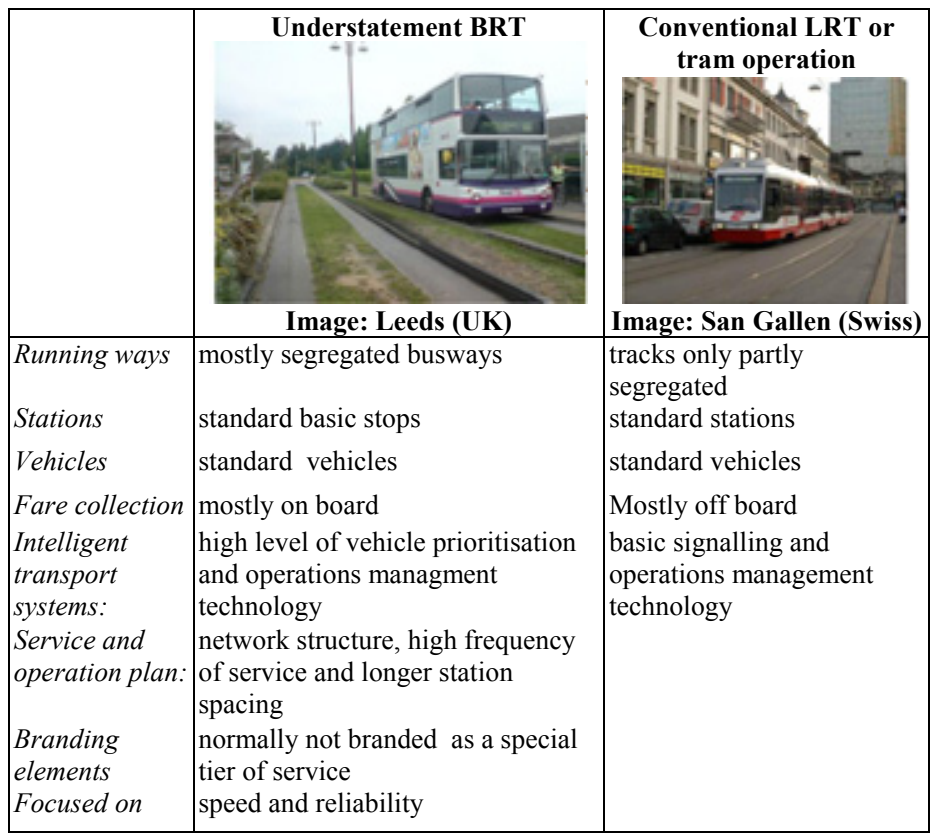

\section{Transport system selection criteria}

The type of public transport system was chosen according to the characteristics of urban mobility, in particular the demand of transport and the actual facility. The main difficulty encountered in implementing a constrained guide mass transport system in a historic town is constituted by the number of restrictions on the surface and the lack of space. Therefore alternate lanes for the BHLS should be provided, regulated by traffic lights, in segments characterized by small cross sections that do not allow the two-way running. From the analysis of supply and demand, the best transport system to realize the connection was the BHLS system that allows to manage traffic flows between a bus and a traditional tram and helps to ensure a good reliability and efficiency with significantly lower costs than those of a tramway (see Table 3) [6]. In fact this transport system can get very low travel time and very high commercial speeds, such as those of LRT. Therefore the BHLS system was preferred to the tram for both economic and technical reasons. From an economic point of view, the BHLS system needs construction and maintenance costs lower than the LRT system.

From a technical point of view, the BHLS system shows the same characteristics in terms of frequency, speed, reliability and efficiency of the LRT but with greater flexibility. 
Table 3: Comparison between costs of BRT and LRT systems.

\begin{tabular}{|l|l|l|}
\hline \multicolumn{1}{|c|}{ Transport mode } & \multicolumn{1}{|c|}{ BRT } & \multicolumn{1}{c|}{ LRT } \\
\hline $\begin{array}{l}\text { Average capital cost (2000 } \\
\text { US\$mile) (millions) }\end{array}$ & 13.46 & 34.70 \\
\hline $\begin{array}{l}\text { Average operation cost (2000 } \\
\text { US\$ per vehicle revenue mile) }\end{array}$ & 4.73 & 12.22 \\
\hline
\end{tabular}

\section{Vehicle technical characteristics}

In this work, the Civis Irisbus of $18 \mathrm{~m}$ length with optical guide was chosen. This vehicle can hold 144 passenger and is equipped with an hybrid engine combining a diesel and an electric motor that allow to obtain lower emissions of pollutants and therefore a lower environmental impact than traditional buses.

The main characteristics of this vehicle are summarized in Table 4.

Table 4: Civis Iribus technical characteristics.

\begin{tabular}{|l|r|l|r|}
\hline Length (mm) & 17900 & beam footprint (mm) & 11750 \\
\hline Pitch (mm) & $5355 / 6675$ & turning radius (mm) & 10247 \\
\hline Max High (mm) & 3300 & projection angle front/rear & $7^{\circ} / 7^{\circ}$ \\
\hline Width (mm) & 2500 & MTT $(\mathrm{Kg})$ & 30000 \\
\hline Overhang front / rear (mm) & $2710 / 3160$ & allowable front $(\mathrm{kg})$ & 7245 \\
\hline Front and rear track (mm) & $2087 / 1869$ & Allowable centre $(\mathrm{kg})$ & 11500 \\
\hline Internal high front/rear (mm) & $2344 / 2108$ & allowable rear $(\mathrm{kg})$ & 12000 \\
\hline Threshold high front/centre/rear (mm) & $320 / 330 / 330$ & & \\
\hline Door width (mm) & 1200 & & \\
\hline
\end{tabular}

\section{Transport demand}

The following components of the demand were analyzed:

- resident demand: are the total daily trips by residents, that was found to be $51393 \mathrm{travel} /$ day;

- commuter for work: are the systematic movements of those who work daily in the urban area and is equal to 8750 travel/day;

- commuter for study: are the systematic movements of those who study daily in the urban area and is equal to 8120 travel/day;

- occasional city users: are the occasional travel daily attracted / generated by urban area that are equal to 1365 travel/day;

- hospital demand: daily trips planned with the complete transfer of the hospital from S. Chiara to Cisanello that are 3600 travel/day. 
Therefore, we considered a modal split as shown in Figure 1 and obtained a potential demand of 15,000 pass/day. Finally, assuming that this demand is divided between the from-and-to journey at 50/50, we have 7500 pass/day for each direction.

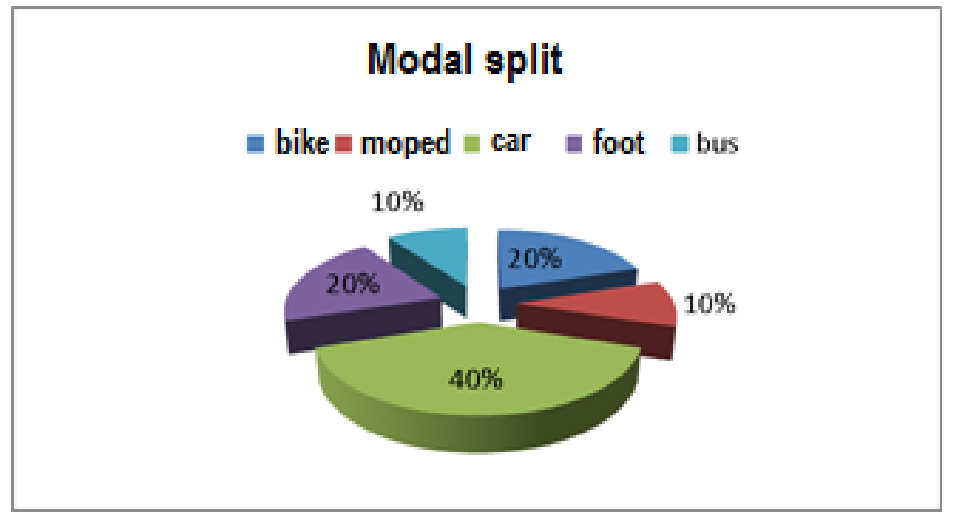

Figure 1: Assumed modal split percentages.

\section{Facility technical features}

The connection in a dedicate lane foreseen in this work has a length of about $3.7 \mathrm{~km}$, there are 3 stops along the way, located in the main attractors point, that have been identified in the Congress Palace, where there are nearby the Faculty of Economics, Agriculture and Veterinary Medicine, and a park and ride; in the area of Mediaworld, where there are many offices, and in the commercial area of Pisanova where the bus stop also ensures a good accessibility to the cemetery of Cisanello and to the new park and ride which will be built nearby.

Along the way there are two short sections of alternating one-way for BHLS regulated by traffic light system.

\section{Features of the line}

Built the outflow model of the new BHLS line and after calculating the travel time, that is composed of the dwell time at the bus stop, that could be calculated using the di Gangi and Nuzzolo model [7], the starting, the ride and the braking times, that could be determined by the constructions of traction diagrams, the various characteristic parameters can be determined:

- commercial speed $(\mathrm{Vc}) 37 \mathrm{Km} / \mathrm{h}$;

- operating speed (Ve) $20 \mathrm{Km} / \mathrm{h}$;

- Lap time (Tg), when considered a switching time of 300 secs at the terminal, allowing BRT to recover any delay, was found to be 22 minutes;

- number of vehicles required on the line $(\mathrm{N})$ during rush hour: 5. In the off peak hours, being characterized by a different demand, less vehicles are required: only 2 . In fact, we choose to decrease the frequency, maintaining 
the same level of service offered in order to make the service more economical, saving a portion of vehicles in the less loaded hours and therefore of drivers in service;

- hourly transport capacity of the line (Cf) 1728 pass / h.

The hourly chart of the line that lists the characteristic parameters of each section is shown in Figure 2.

\section{Signalized roundabouts}

The realization of a line with the described characteristics can be achieved in two different ways: important facilities can be built in order to physically separate the path of the BHLS from that of private vehicles (i.e. underpasses, new bridges) so that the two opposite flows do not interfere each one with the other when crossing intersections; alternatively, traffic regulation systems can be used, providing signalized roundabouts, with traffic light priority to the bus, in which the crossing of the BHLS is scheduled on one of the branches of the roundabout to avoid to block the overall running, or in its central area.

So, in order to design a signalized roundabout, first of all the branches lanes must be specialized in order to block only the maneuvers in conflict with the BHLS crossing.

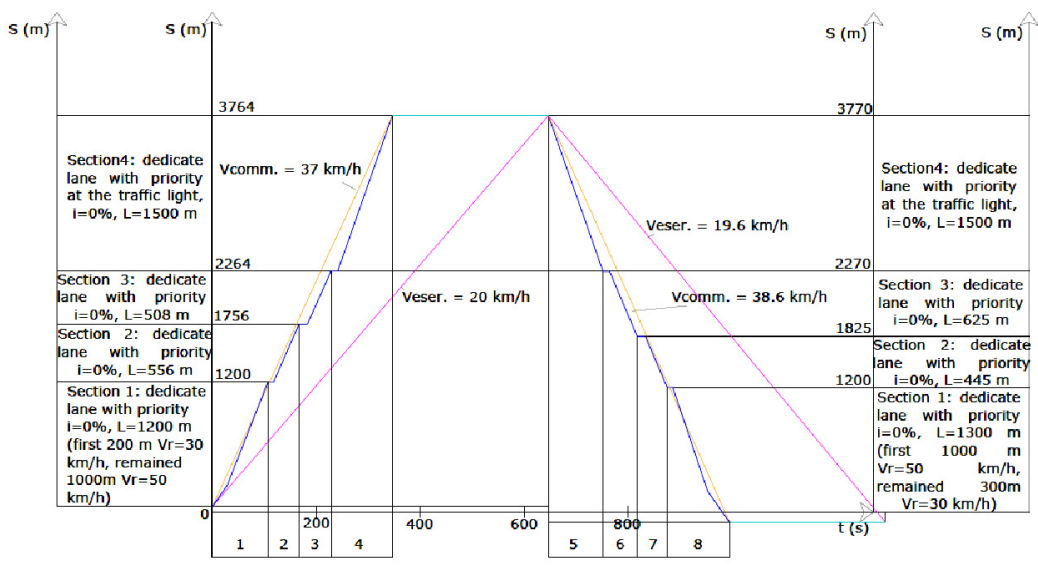

Figure 2: BHLS line hourly chart.

In order to check the intersection operation and to verify that the private car traffic does not suffer excessive penalties two types of tests were performed. The first type concerns the checking of the roundabout in its conventional running, which was performed using the method of SETRA [8] for big roundabouts with the external diameter greater than $40 \mathrm{~m}$, and the CETUR [9, 10] method for smaller ones. The other type regards the checking of the traffic signal that was performed using the program HCS+. 
The traffic light systems are semi actuated, and vehicles in the roundabout carriageway have always green except when the BRT crosses the intersection activating the detectors, placed at $100 \mathrm{~m}$ from it, and stopping all the conflicting flows.

Then, considering the peak hour, when every 5 minutes a bus crosses the intersection in one direction, the traffic light cycle 2.5 minutes long was assumed with amber time for the bus of 3 secs and the green time that depends on the time requested to cross the intersection. The amber time for the vehicle could be assumed 4 secs and the green time is obtained consequentially.

All the tests summarized in Table 5, Table 6 and Table 7 clearly show that the obtained level of service for these solutions is good.

Table 5: Commercial speed, operating speed, travel time in each sections.

\begin{tabular}{|c|c|c|c|c|c|c|c|}
\hline Sec. N. & Vr & Vcomm & ttravel & Sec. N. & Vr & Vcomm & Ttravel \\
\hline 1 & 33.60 & 33.6 & 107.06 & 5 & 51.0 & 51.0 & 105.3 \\
\hline 2 & 33.65 & 28.5 & 48.60 & 6 & 42.6 & 35.3 & 52.8 \\
\hline 3 & 40.00 & 29.7 & 45.70 & 7 & 38.1 & 27.7 & 42.0 \\
\hline 4 & 51.00 & 45.7 & 105.30 & 8 & 33.5 & 29.9 & 107.6 \\
\hline
\end{tabular}

Table 6: Roundabouts traditional check list.

\begin{tabular}{|l|c|c|c|}
\hline Roundabout & Method & Ew $(s)$ & LoS(Int.) \\
\hline Cisanello & SETRA & 15.6 & B \\
\hline Pisanova & SETRA & 10.5 & A \\
\hline Mediaworld & Cetur, Kimber Hollis & 7.6 & A \\
\hline P.za Politeama Pisano & Cetur, Kimber Hollis & 12 & B \\
\hline P.za Guerrazzi & Cetur, Kimber Hollis & 15.2 & B \\
\hline
\end{tabular}

Table 7: Traffic lights check list.

\begin{tabular}{|l|c|c|c|}
\hline Roundabout & Stoppage (s) & Ew (s) & LoS (Int.) \\
\hline Cisanello & 10 & 2.0 & $\mathrm{~A}$ \\
\hline Pisanova & 12 & 4.2 & $\mathrm{~A}$ \\
\hline Mediaworld & 15 & 2.0 & $\mathrm{~A}$ \\
\hline P.za Politeama Pisano & 10 & 2.4 & $\mathrm{~A}$ \\
\hline
\end{tabular}




\section{Concluding remarks}

In this paper, we showed that the use of traffic control systems to manage the roundabout intersections during the passage of BHLS, is capable of ensuring excellent levels of service in mixed traffic, which does not undergo excessive penalties, and allows to create lines characterized by high performance, because of low delays at signalized intersections thanks to the controlled priority.

Moreover, by using traffic light control systems, physical devices to separate the two opposing flows are avoided, which are too costly and very difficult to achieve due to worldwide well-known bad geotechnical characteristics of soils in the whole area of Pisa (e.g., soil under the Leaning Tower!).

\section{References}

[1] http://ec.europa.eu/research/horizon2020.

[2] Gini S. et al., Priority System and Information Workflow for BHLS Services: An Interregional Project Between Italy and Germany, Advanced Microsystems for Automotive Applications 2010, VDI-Buch, 2010, pp. 387-398.

[3] Wright, L., W. Hook, Bus Rapid Transit Planning Guide, 3rd ed., Institute for Transportation \& Development Policy, New York, 2007.

[4] CERTU, Buses with a high level of service (French Bus Rapid Transit): choosing and implementing the right system, 2009.

[5] Sorg D., BRT systems and beyond. Exploring the limits of a popular and rapidly growing urban transport system, Master Thesis ETH, 2011.

[6] Deng T., Nelson J. D., Recent Development in Bus Rapid Transit: A review of the literature, Transport Reviews, 31:1, pp. 69-96, 2011.

[7] Nuzzolo A., M. di Gangi, Una procedura di simulazione per la valutazione del livello di servizio di una linea di trasporto collettivo. In "Models and methods for decision support", AIRO, 1990.

[8] Louah G., Capacite des carrefours giratoires interurbains. Documentation Technique 44. Ministere de l'Equipement du Logement, de l'Amenagement du Territoire et des Transports, Paris, 1987.

[9] Pratelli, A., Design of modern roundabouts in urban traffic systems, in Brebbia \& Dolezel (eds.), Urban Transport XII, WIT Press, pp. 83-93, 2006.

[10] Kimber R. M. and E. M. Hollis, Traffic queues and delays at road junctions. TRRL Laboratory Report LR 909. Crowthorne, England, 1979. 